\title{
An In Silico Analysis Identified FZD9 as a Potential Prognostic Biomarker in Triple-Negative Breast Cancer Patients
}

(1) Daniel Rodrigues de Bastos ${ }^{1}$, (1) Mércia Patrícia Ferreira Conceiçãa ${ }^{1}$, (D) Ana Paula Picaro Michelli²,

(10) Jean Michel Rocha Sampaio Leite 3 , (1) Rafael André da Silva $a^{4}$, (10) Ricardo Cesar Cintra 5 , (1) Jeniffer Johana Duarte Sanchez

(D) Cesar Augusto Sam Tiago Vilanova-Costa ${ }^{7}$, (1) Antonio Márcio Teodoro Cordeiro Silva ${ }^{8}$

${ }^{1}$ Department of Oncology, Universidade de São Paulo, São Paulo, Brazil

${ }^{2}$ Department of Biological Sciences, Thyroid Molecular Science Laboratory, Universidade Federal de São Paulo, São Paulo, Brazil

${ }^{3}$ Department of Nutrition, Universidade de São Paulo, Faculty of Public Health, São Paulo, Brazil

${ }^{4}$ Department of Cellular \& Developmental Biology, Universidade de São Paulo, Institute of Biomedical Sciences, São Paulo, Brazil

${ }^{5}$ Department of Biochemistry, Universidade de São Paulo, Institute of Chemistry, São Paulo, Brazil

${ }^{6}$ Department of Statistics and Applied Math, Universidade Federal do Ceará, Fortaleza, Brazil

${ }^{7}$ Laboratory of Tumor Biology and Oncogenetics Hospital, Araujo Jorge, Goiânia, Brazil

${ }^{8}$ Department of Medicine, Pontifícia Universidade Católica de Goiás, School of Medical Sciences, Biomedics and Pharmaceuticals, Goiânia, Brazil

\begin{abstract}
Objective: Breast cancer (BC) is the main cause of cancer-related deaths in women across the world. It can be classified into different subtypes, including triple-negative (TN), which is characterized by the absence of hormone receptors for estrogen and progesterone and the lack of the human epidermal growth factor receptor 2. These tumors have high heterogeneity, acquire therapeutic resistance, and have no established target-driven treatment yet.

The identification of differentially expressed genes in TN breast tumors and the in silico validation of their prognostic role in these tumors.

Materials and Methods: We employed a microarray dataset and, by using the GEO2R tool, we identified a list of differentially expressed genes. The in silico validation was conducted using several online platforms including the KM Plotter, cBioPortal, bc-GenExMiner, Prognoscan, and Roc Plotter.

Results: We observed that FZD9 was among the top differentially expressed genes in a cohort of patients with different TNBC subtypes. The FZD9 expression was significantly different in TN breast tumors than in non-TN (nTN) breast tumors $(\mathrm{p}<0.0001)$, and the basal TN subtype showed the highest levels $(\mathrm{p}<0.0001)$. In addition, the FZD9 levels were significantly inversely and positively proportional $(\mathrm{p}<0.0001)$ to estrogen receptor, progesterone receptor, and human epidermal growth factor receptor- 2 clinical parameters. The high levels of FZD9 were associated with worse overall survival ( $\mathrm{p}=0.007$ ), relapse-free survival ( $\mathrm{p}=5.8 \mathrm{e}-05)$, and worse survival in patients who received chemotherapy ( $\mathrm{p}=3.2 \mathrm{e}-05 ; 0.007)$.

Conclusion: Our cumulative results demonstrated that FZD9 plays an important role in TNBC and may be a potential prognostic biomarker. Nevertheless, further in vitro and in vivo assays are necessary to confirm our findings and to strengthen the evidences about the mechanisms by which FZD9 functions in these tumors.
\end{abstract}

Keywords: FZD9, breast cancer, triple-negative breast cancer, in silico analysis, biomarkers

Cite this article as: de Bastos DR, Ferreira Conceiçáo MP, Picaro Michelli AP, Leite JMRS, da Silva RA, Cintra RC, Duarte Sachez JJ, Vilanova-Costa CAST, Silva AMTC. An In Silico Analysis Identified FZD9 as a Potential Prognostic Biomarker in Triple-Negative Breast Cancer Patients. Eur J Breast Health 2021; 17(1): 42-52.

\section{Introduction}

Breast cancer (BC) is the main cause of cancer-related deaths of the world's female population as well as, particularly the Brazilian women (1). The National Cancer Institute in Brazil (INCA) estimated 66,280 new BC cases in 2020, comprising 29.7\% of all tumors with a stratified primary location; this estimate is much higher than that for the cancer of the colon and rectum $(9.2 \%$ of all cases) and cervical cancer $(7.4 \%)$ in women.

$\mathrm{BC}$ tumors can be categorized into five main subtypes that have been widely discussed in the literature according to the PAM50 classification: Basal (B), Luminal A (LA), Luminal B (LB), human epidermal growth factor receptor-2+ (HER2+), and normal breast-like (N). Another important classification encompasses triple-negative (TN) and non-TN (nTN) breast tumors, which are identified based on the immunohistochemistry outcomes for the hormone estrogen receptor (ER) and progesterone receptor (PR), and by the amplification of the HER2 $(2,3)$. The lack of expression of these three important membrane receptors classify them as TN (4). Approximately $80 \%$ of all basal tumors can be classified as TN, with similar expression profiles between these two classes $(5,6)$. 
In contrast to nTN tumors, TN tumors present with low survival, lack therapeutic targets, and have a high relapse rate and a high metastatic potential. They are also highly heterogeneous, and sub-classified in six distinct groups: basal-like 1 (BL1), basal-like 2 (BL2), mesenchymal (M), mesenchymal stem-like (MSL), luminal androgen receptor (LAR), and immunomodulatory (I) (7), which poses a challenge to discover new therapeutic targets in order to provide more effective treatments for the patients.

Among the components underlying tumorigenesis, the FZD family members participate in both canonical and non-canonical Winglesstype (Wnt) pathways, which have been strongly implicated in tumor invasion and progression. The FZD family is responsible for coding transmembrane proteins with the protein receptor domains of Wnt signaling, which in turn is comprised of canonical or Wnt $/ \beta$-catenindependent and the non-canonical or Wnt $\mid \beta$-catenin-independent signals. These protein receptor domains activate target genes involved in several biological processes such as embryonic and organ development, homeostasis, cell proliferation, self-renewal, differentiation, and migration. In addition, they have been implicated in tumorigenesis, cell invasion, tumor malignancy, and survival (8-10). In addition, the upregulation of FZD members has been reported in some cancers, including gastric and renal cell carcinoma, which suggests their direct involvement in carcinogenesis $(11,12)$.

In this context, we employed the microarray dataset GSE76275 and performed in silico analysis to identify the potential prognostic biomarkers and discover new therapeutic targets in TN breast tumors (13). Our preliminary analysis revealed that the mRNA frizzled class receptor 9 (FZD9) is differentially expressed in TN tumors. We confirmed the reproducibility and reliability of this finding by validating it on a larger public dataset and found that FZD9 is differentially expressed across the TN subtypes and is associated with low survival, tumor recurrence, and tumor grade. Taken together, our results suggest that FZD9 is a promising transcript and a potential biomarker in the study of these tumors.

\section{Materials and Methods}

\section{Geo database-data access}

The dataset of the published online microarray GSE76275 was accessed through the platform GEO (ncbi.nlm.nih.gov/geo/) and analyzed using the online tool GEO2R (ncbi.nlm.nih.gov/geo/geo2r) (13). The criteria of gene selection for further analysis was the adjusted p-value by Benjamini and Hochberg (False Discovery rate) of $<0.001$ and biological relevance (Figure 1).

\section{Expression analysis}

The Breast Cancer Gene-Expression Miner v4.4 (bc-GenExMiner v4.4) (bcgenex.centregauducheau.fr/BC-GEM/GEM-requete.php) is an online mining tool of transcriptomic data of properly annotated BC $(14,15)$. We used the RNA-seq data to analyze the FZD9 expression with clinical parameters such as ER, PR, HER-2, and different clinical BC subtypes.

\section{Survival analysis}

The prognostic role of FZD9 was analyzed by using the Kaplan-Meier Plotter (kmplot.com/analysis/) to create the overall survival (OS) and relapse-free survival (RFS) curves (16). The FZD9 expression in patients with $\mathrm{BC}$ was classified as either high or low based on its median expression level. Only a validated probe was selected based on the automatic best cut-off value selection criteria.

\section{cBioPortal data}

cBioPortal (https://cbioportal.org) is an online and multi-functional database that contains gene expression and other features of different types of cancer sourced from various studies $(17,18)$. In the present work, we accessed the FZD9 expression of 1,108 cases with RNA seq V2 RSEM data from the Firehouse dataset. The clinical information was cross-referenced with quantitative and qualitative expression data for associations and correlation statistics.

\section{ROC Plotter analysis}

ROC Plotter is a user-friendly online tool (19). With transcriptomic data from 3,104 BC patients treated and untreated with endocrine therapy, anti-HER2 therapy, or chemotherapy, we quickly assessed the pattern of expression of genes of interest in the face of the treatment received by the patient.

\section{Statistical analysis}

All data were evaluated for Gaussian distribution, and the t-test or Mann-Whitney U test was performed to assess the differences between the two groups. Kruskal-Wallis was applied for the analysis of three or more groups, followed by the post-hoc Dunn's test. The results were considered statistically significant at $\mathrm{p}<0.05$ or, whenever necessary, according to adjusted p-values. Pearson's correlation analyses between several genes were also performed. All statistical analysis was performed

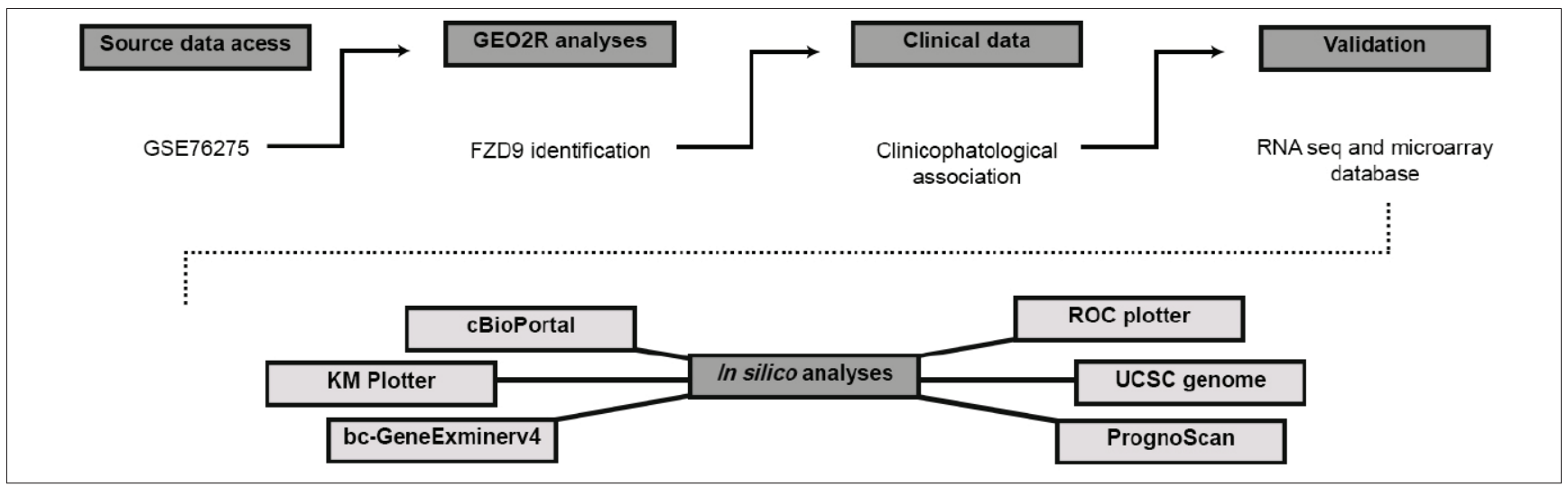

Figure 1. Methodological design depicting the study protocol and the main databases used for identification, in silico analysis, and the validation of FZD9 
in the Statistical Package for Social Sciences (SPSS; version 25). A forest plot and other graphs were constructed in the RStudio v.1.0.153 and GraphPad Prism v. 7 (California, USA), respectively.

\section{Results}

\section{Geo database}

The GSE76275 dataset was derived from the study of Burstein et al. (13) that aimed to identify new targets in different TNBC subtypes. The expression profile of 265 breast tumor samples, 198 of which were classified as TN and 67 as n-TN tumors, was evaluated. The present study divided these samples into two large groups: TN and n-TN, and identified a list with 54,675 probes using the online tool GEO2R (S1 Table), with FZD9 being the seventh probe with the lowest adjusted p-value (Figure 2a).

In order to address the role of this transcript in TNBC, we compared its expression in TN and n-TN tumors and also across different TN subtypes. We noted a significant increase in the FZD9 expression in patients with TN tumors than in those with n-TN tumors (adjusted $\mathrm{p}<0.0001$; Figures $2 \mathrm{~b}, \mathrm{c})$. Considering the different subtypes of TNBCs, we observed that basal tumors had higher mean levels of FZD9 expression (Figure 2d).

In addition, we observed significant associations between FZD9's low and high expression categories and the tumor status (TN vs n-TN; $\mathrm{p}<0.0001)$, tumor grade $(\mathrm{p}<0.0001)$, and also regarding the $\mathrm{TN}$ subtypes identified in the analyzed cohort $(\mathrm{p}<0.0001$; Table 1$)$.

In the analysis conducted on bcGenExMiner, we identified significant mean differences in a larger cohort, which confirmed our conclusions displayed in Table 1 (Table 2). In addition, we identified mean high levels of FZD9 expression in patients with p53 mutations $(\mathrm{p}<0.0001)$, grade 3 of the Scarff-Bloom-Richardson (SBR) classification $(\mathrm{p}<0.0001)$, and also in Nottingham Prognostic Index $(\mathrm{p}<0.0001)$. Patients with basal-like status, TN status, and the combination of TN and basal-like status exhibited a mean high expression level of FZD9 $(\mathrm{p}<0.0001)$ (Table 2).

\section{FZD9 expression analysis}

An analysis of the expression pattern of FZD9 in breast tumors was conducted on the bc-GenExMiner portal, and significant mean differences were noted regarding hormone receptors $(\mathrm{p}<0.0001)$ and HER2 ( $<<0.0001)$ (Figures 3a-c).

Important mean differences were recorded on the bc-GenExMiner. The mean FZD9 expression pattern was significantly higher in basal tumors than in other subtypes as per the PAM50 classification (Figure $3 \mathrm{~d})$. In relation to the histopathological characteristics, invasive ductal carcinoma presented with a high mean expression of FZD9 (Figure 3e). Finally, women with p53 mutation (Figure 3f) and those aged $<51$ years (Figure 3g) also showed higher FZD9 expression.

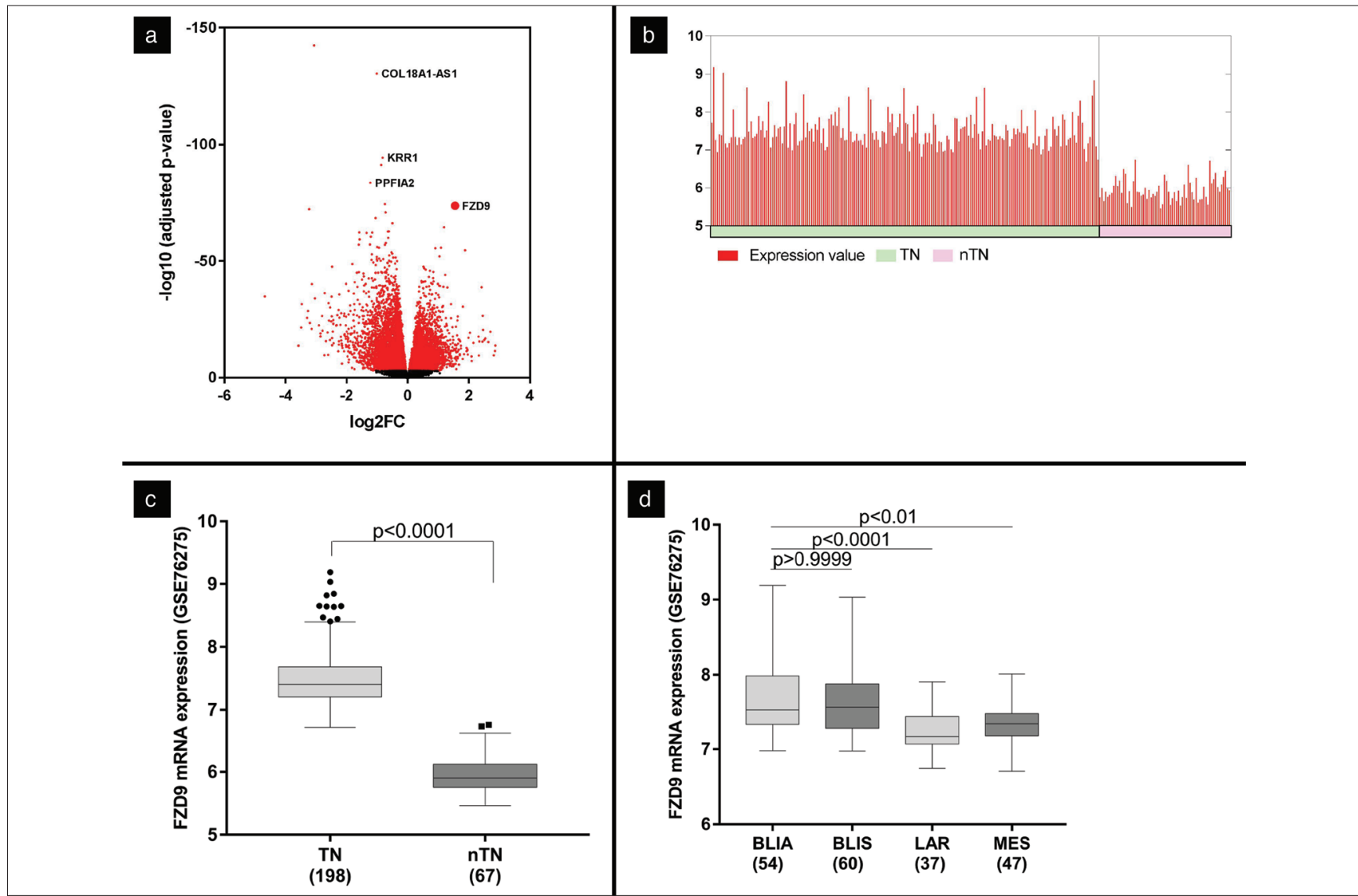

Figure 2. a) Volcano plot containing the probes identified in the microarray data set. FZD9 was selected for in silico validation because it had the top adjusted p-value and factual characteristics; b) Representative image indicating the levels of FZD9 expression across breast tumors samples; c) The FZD9 expression in triple and non-triple negative patients; d) The FZD9 expression in different subtypes of triple negative breast cancer samples

TN: Triple negative; n-TN: Non-triple negative; BLIA: Basal-like Immune-activated; BLIS: Basal-like Immune-suppressed; LAR: Luminal androgen receptor; MES: Mesenchymal 
Table 1. Clinical-pathological characteristics of patients with triple negative and non-triple negative breast cancer derived from the GEO database GSE76275, and association with FZD9 expression

\section{Parameters}

Age

$\leq 40$

$>40 \leq 70$

$>70$

Race

Asian

Asian/Pacific islander

Caucasian

Menopausal

Post

Pre

Histology

Adenocarcinoma/carcinoma

IDC

ILC

Other breast cancer

Stage

II

III

IV

\section{TN status}

not TN

TN

Tumor grade

Moderately differentiated

Poorly differentiated

Well differentiated

Tumor size

$\leq 2 \mathrm{~cm}$

$>5 \mathrm{~cm}$

$2-5 \mathrm{~cm}$

Any size with direct extension

\section{TN subtype}

Basal-like immune-activated (BLIA)

Basal-like immune-suppressed (BLIS)

Luminal-AR (LAR)

Mesenchymal (MES)

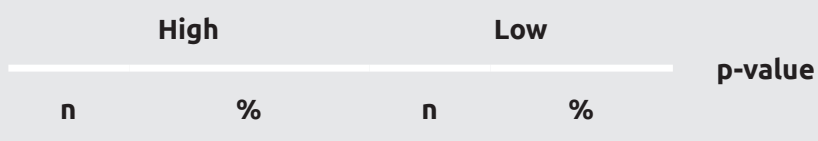

$\begin{array}{llll}17 & 13.4 & 12 & 9.3 \\ 93 & 73.2 & 99 & 76.7 \\ 17 & 13.4 & 18 & 14.0\end{array}$

$\begin{array}{llll}2 & 1.6 & 1 & 0.8\end{array}$

$4 \quad 3.1$

$122 \quad 95.3$

$\begin{array}{ll}0 & 0.0\end{array}$

0.051

$6 \quad 5.5$

$64 \quad 58.2$

$132 \quad 99.2$

40.36 .4

$15 \quad 13.0$

$67 \quad 58.3$

0.106

$40 \quad 36.4$

$\begin{array}{ll}33 & 28.7\end{array}$

$2 \quad 1.5$

$5 \quad 7.6$

$127 \quad 96.2$

$1 \quad 0.8$

$2 \quad 1.5$

$61 \quad 92.4$

$\begin{array}{ll}0 & 0.0\end{array}$

$\begin{array}{ll}0 & 0.0\end{array}$

14

$46 \quad 51.1$

$29 \quad 32.2$

1.1

$\begin{array}{ll}0 & 0.0\end{array}$

132

100.0

$4 \quad 4.5$

$\begin{array}{ll}56 & 62.9\end{array}$

$28 \quad 31.5$

0.055

0.107

$$
0.0
$$

$1 \quad 1.1$

$67 \quad 50.4$

$66 \quad 49.6$

$<0.0001$

$\begin{array}{llll}28 & 24.8 & 51 & 52.0\end{array}$

$\begin{array}{llll}84 & 74.3 & 43 & 43.9\end{array}$

$<0.0001$

$\begin{array}{llll}1 & 0.9 & 4 & 4.1\end{array}$

$27 \quad 20.9$

$8 \quad 12.1$

$\begin{array}{ll}6 & 4.7\end{array}$

$92 \quad 71.3$

$\begin{array}{ll}6 & 9.1\end{array}$

$47 \quad 71.2$

0.154

$4 \quad 3.1$

$5 \quad 7.6$

$\begin{array}{ll}44 & 33.3 \\ 46 & 34.8 \\ 12 & 9.1 \\ 30 & 22.7\end{array}$

$10 \quad 15.2$

$14 \quad 21.2$

$25 \quad 37.9$

$<0.0001$ 
Table 2. Relationship between FZD9 expression and clinical parameters of breast cancer patients using the bc-GenExMiner database

\begin{tabular}{|c|c|c|c|c|c|c|}
\hline Variables & Patient number & FZD9 RNA seq & p-value* & Patient number & FZD9 microarray & p-value* \\
\hline \multicolumn{7}{|l|}{ Age } \\
\hline$\leq 40$ & 239 & - & \multirow{3}{*}{$<0.0001$} & 797 & - & \multirow{3}{*}{$<0.0001$} \\
\hline$>40 \leq 70$ & 2,851 & Decreased & & 5,292 & Decreased & \\
\hline$>70$ & 1,217 & Decreased & & 1,417 & Decreased & \\
\hline \multicolumn{7}{|l|}{ ER } \\
\hline Negative & 551 & Increased & \multirow{2}{*}{$<0.0001$} & 2,249 & \multirow{2}{*}{$\begin{array}{c}\text { Increased } \\
-\end{array}$} & \multirow{2}{*}{$<0.0001$} \\
\hline Positive & 3,911 & - & & 6,310 & & \\
\hline \multicolumn{7}{|l|}{ PR } \\
\hline Negative & 828 & Increased & \multirow{2}{*}{$<0.0001$} & 1,427 & \multirow{2}{*}{$\begin{array}{c}\text { Increased } \\
-\end{array}$} & \multirow{2}{*}{$<0.0001$} \\
\hline Positive & 3,498 & - & & 1,994 & & \\
\hline \multicolumn{7}{|l|}{ HER2 } \\
\hline Negative & 3,582 & Increased & \multirow{2}{*}{$<0.0001$} & 2,387 & - & \multirow{2}{*}{0.0955} \\
\hline Positive & 661 & - & & 436 & - & \\
\hline \multicolumn{7}{|l|}{ P53 status } \\
\hline Wild type & 699 & - & \multirow{2}{*}{$<0.0001$} & 1,328 & - & \multirow{2}{*}{$<0.0001$} \\
\hline Mutated & 328 & Increased & & 652 & Increased & \\
\hline \multicolumn{7}{|l|}{ Nodal status } \\
\hline Negative & 2,415 & Increased & \multirow{2}{*}{0.0105} & 4,431 & - & \multirow{2}{*}{0.2060} \\
\hline Positive & 1,646 & - & & 3,458 & - & \\
\hline \multicolumn{7}{|l|}{ SBR } \\
\hline 1 & 544 & - & \multirow{3}{*}{$<0.0001$} & 889 & - & \multirow{3}{*}{$<0.0001$} \\
\hline 2 & 1,699 & Decreased & & 2,926 & Decreased & \\
\hline 3 & 1,374 & Increased & & 2,933 & Increased & \\
\hline \multicolumn{7}{|l|}{ NPI } \\
\hline 1 & 1,173 & - & \multirow{3}{*}{$<0.0001$} & 1,234 & - & \multirow{3}{*}{$<0.0001$} \\
\hline 2 & 1,525 & Increased & & 2,119 & Increased & \\
\hline 3 & 416 & Increased & & 675 & Increased & \\
\hline \multicolumn{7}{|l|}{ Basal-like status } \\
\hline Non basal-like & 3,836 & - & - ח & 7,231 & - & \\
\hline Basal-like & 832 & Increased & $<0.0001$ & 1,870 & Increased & $<0.0001$ \\
\hline Triple-negative stat & & & & & & \\
\hline Non triple-negative & 4,119 & - & $<\cap \cap \cap 01$ & 6,590 & - & $<0 \cap 0001$ \\
\hline Triple-negative & 317 & Increased & $<0.0001$ & 572 & Increased & $<0.0001$ \\
\hline Triple-negative and & asal-like status & & & & & \\
\hline $\begin{array}{l}\text { Not basal-like and } \\
\text { not TNBC }\end{array}$ & 3,689 & - & $<0.0001$ & 5,811 & - & $<0.0001$ \\
\hline Basal-like and TNBC & 267 & Increased & & 406 & Increased & \\
\hline
\end{tabular}

SBR: Scarff-Bloom-Richardson; NPI: Nottingham Prognostic Index; TNBC: Triple-negative breast cancer; ER: Estreogen receptor; PR: Progesterone receptor; HER2: Human epidermal growth factor receptor-2 *Statistical significance was determined by the Welch's test. SBR | NPI: p-value refers to the group and the level of higher expression is reported in relation to level 1

\section{Expression of FZD9 in basal tumors, TNBCs, and survival}

Based on information obtained from Prognoscan, we identified 5 cohorts showing an association between FZD9 and worse prognosis
FZD9 expression in basal tumors and in those classified as TN, which suggested that both the tumors presented with higher mean mRNA levels (Figures 4b, c). Using the online tool KM Plotter, we identified a significant difference in the survival between high and lower levels 
of FZD9. Patients with high levels of FDZ9 showed poor prognosis (Figures $4 \mathrm{~d}, \mathrm{e}$ ), this difference was more pronounced in the basal tumor group (Figures 4f, g).

\section{Mutational profile and co-expression analysis}

We observed an $8 \%$ frequency of alterations in FZD9 using RNAseq data from the Firehose-cBioPortal databank (Figure 5a). In the same bank, we conducted a correlation analysis, and a total of 20,186 transcripts were identified with multiple Pearson's Correlation values and q-value (adjusted p-value). The 6 genes with the highest adjusted p-values and Spearman's correlation are highlighted in Figure 5b. Under significant correlations, FZD9 and the top 6 genes showed a methylation pattern that was directly proportional to the expression profile, considering the categories TN and n-TN (Figure 5c), which suggests that epigenetic alterations are the main mechanism active in TN tumors.

Figure $5 \mathrm{~d}$ shows the correlations for patients with basal tumors. The correlations among the variables considered were high, either positive or negative. In particular, FDZ9 showed a high positive correlation with RGMA, YBX1, and HAPLNS and a high negative correlation with FOXA1, XEP1, and ESR1 (Figure 5d). The correlations for TNBC patients are shown in Figure 5e. The correlations were highly positive between FOXA1 and XEP1 and highly negative with XEP1.

The following analysis revealed that basal tumors and/or TN tumors have an expression pattern distinct from those of the other tumor subtypes. After classifying tumors as $\mathrm{TN}$ and $\mathrm{n}$-TN based on the immunohistochemical data about the hormone receptors and HER2, an analysis revealed high mean expression of FZD9 in the group of patients with TN tumors (Figure 6a). With reference to the PAM50 classification, we observed higher FZD9 mean expression levels in basal tumors (Figure 6b). The methylation pattern in the basal tumors and the PAM50 classification corroborates coherently with the expression levels of FZD9 in these tumors (Figures 6c, d). Figure 6e depicts the Pearson correlations between FDZ9 and Wnt variables; there was no evidence of strong linear correlations between them.

\section{Survival according to treatment}

Considering the reports of several past studies indicating FZD9 as a potential biomarker for the treatment response to radiotherapy and chemotherapy, we conducted a survival analysis using the KM Plotter while considering only those patients who were treated with chemotherapy (20). We found that both the RFS (Figures 7a, b) and OS (Figures $7 \mathrm{c}, \mathrm{d}$ ) exhibited a significant worse prognosis in the group of patients with high FZD9 levels, which is even more striking for basal tumors. There was a significant difference in the median FZD9 expression levels between TNBC patients with no response to chemotherapy treatment when compared to those who responded (Figure 7e). In agreement with this result, the analysis to classify patients between respondent and non-respondent groups based on the RFS at 5 years showed a subtle significant association, as evidenced by the outcomes of the area under the curve (AUC), True Positive Rate, and lower False Positive Rate (FPR) (Figure 7f). In addition, we found a significant evidence supporting an association between the variables FDZ9 and tumor recurrence (see Figure 7g).

\section{Discussion and Conclusion}

TNBC has gained visibility since it has a poor prognosis and lacks molecular targets for the development of effective therapies. Wnt signaling has been associated with worse prognosis and reduced OS in these tumor types, which was proved by the high levels of $\beta$-catenin expression $(21,22)$.

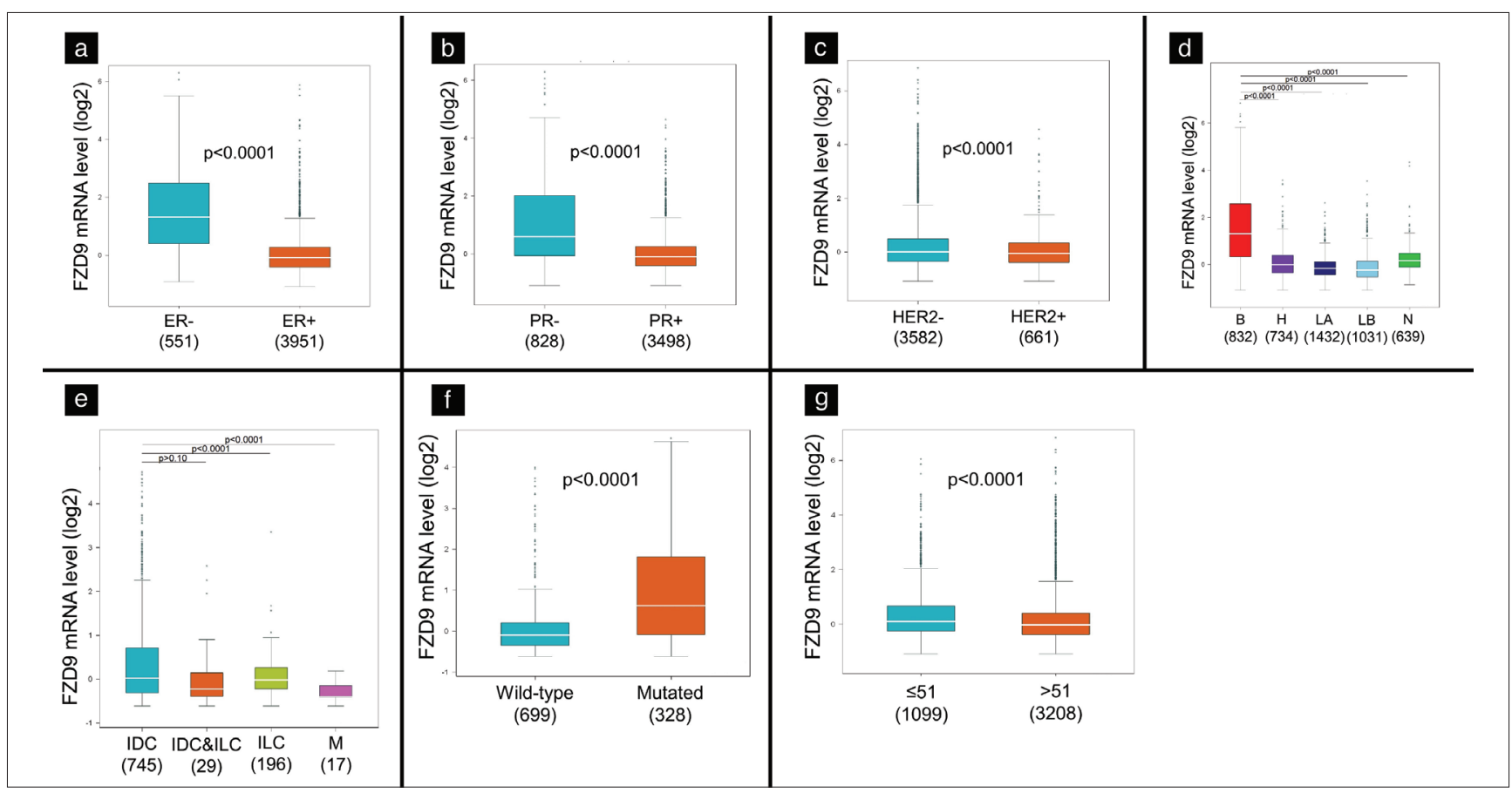

Figure 3. The expression pattern of FZD9 mRNA according to different clinical parameters using the bc-GenExMiner software. Analyses is shown for a) estrogen receptor, b) progesterone receptor, c) HER2, d) molecular subtypes, e) breast cancer histological subtypes, f) p53 mutational status, and g) age. Only RNA seq 


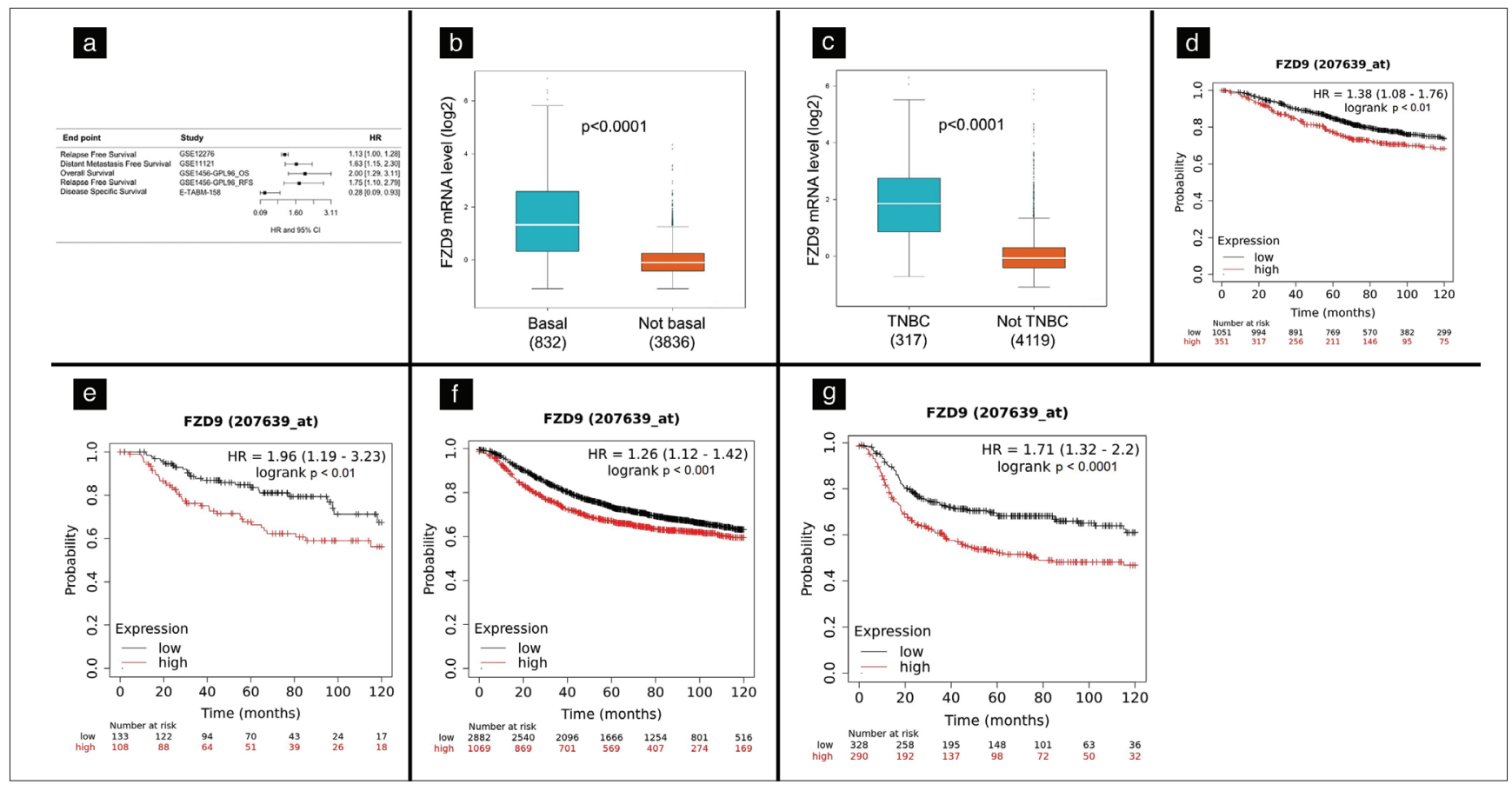

Figure 4. Kaplan-Meier curves and forest plot evaluating the prognostic value of FZD9 in breast cancer patients using KM plotter and Prognoscan a) Forest Plot based on the FZD9 Prognoscan analysis. Only breast cancer dataset with cox p<0.05 were considered. The expression pattern of the FZD9 mRNA as a function of $\mathbf{b}$ ) the basal subtype versus non-basal subtype and c) TNBCs and n-TNBCs tumors. Overall survival analysis $\mathbf{d}$ ) considering all subtypes of breast tumors and $\mathbf{e}$ ) basal tumors. The analysis of recurrence-free survival showing $\mathbf{f}$ ) all tumor subtypes and $\mathbf{g}$ ) basal subtypes

TNBC: Triple-negative breast cancer

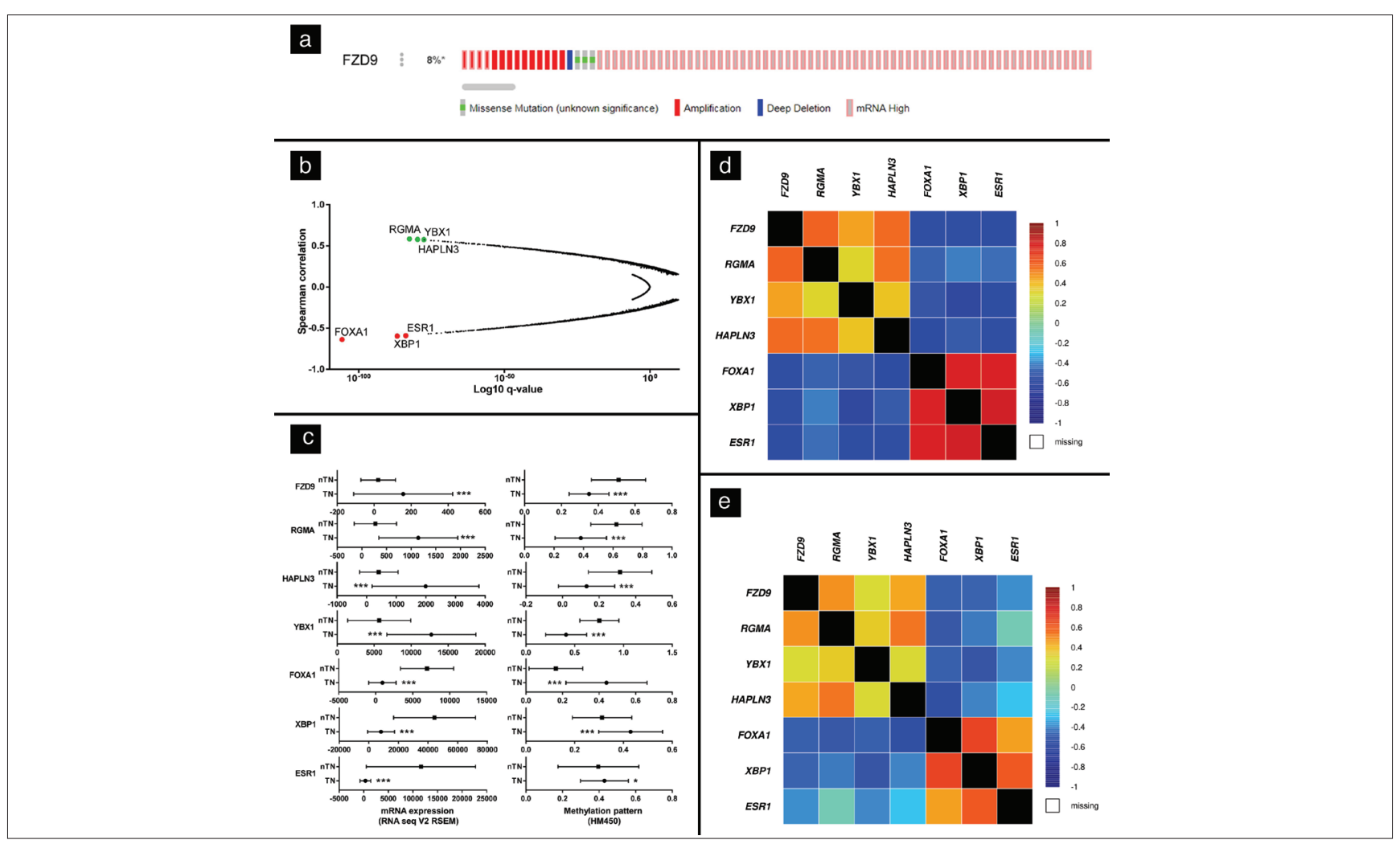

Figure 5. a) Profile of changes in the FZD9 expression in patients with breast tumors through the cBioPortal; b) The correlation test in cBioPortal showing 20,186 transcripts. Six with the highest $r$ and corrected p-value (q-value) are shown in the graph; c) Data downloaded from cBioPortal, and TN or n-TN FZD9 mRNA expression analysis in the left column and methylation patterns in the right column; Correlation analysis conducted on bc-GenExMiner in d) basal and e) TNBC patients 


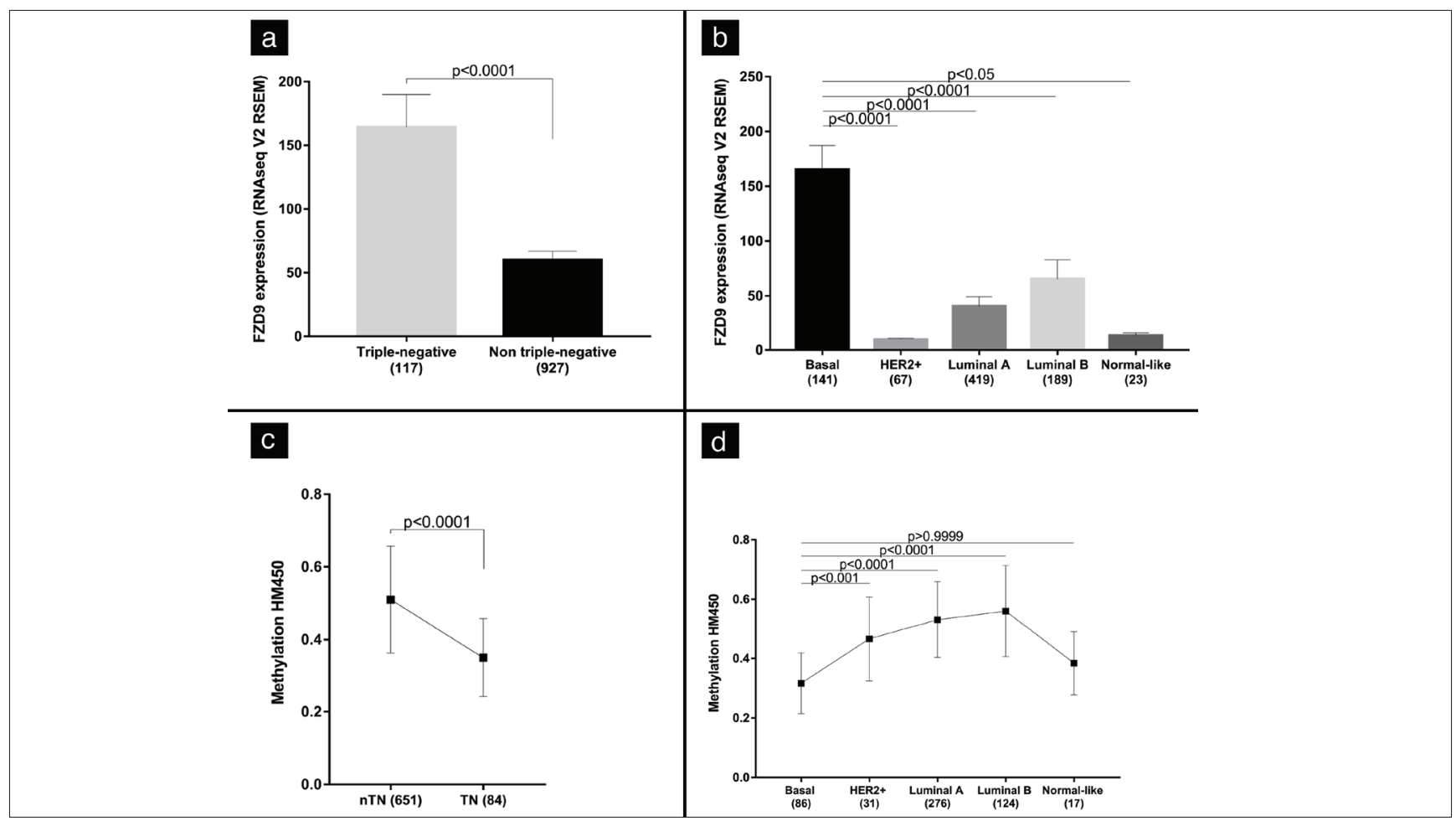

Figure 6. a) The FZD9 mRNA expression is significantly higher in TN patients; b) Multiple comparison testing showing the basal subtype and its greatest expression in relation to the other tumor subtypes; Methylation profile of FZD9 in c) TN or n-TN and d) PAM50 classification; e) Pearson's linear correlation coefficients of the variable FDZ9 with the variables Wnt2, Wnt3A, Wnt3, Wnt5A, Wnt7A, and MKI67. All data are downloaded from the Firehose on cBioPortal database

TN: Triple-negative

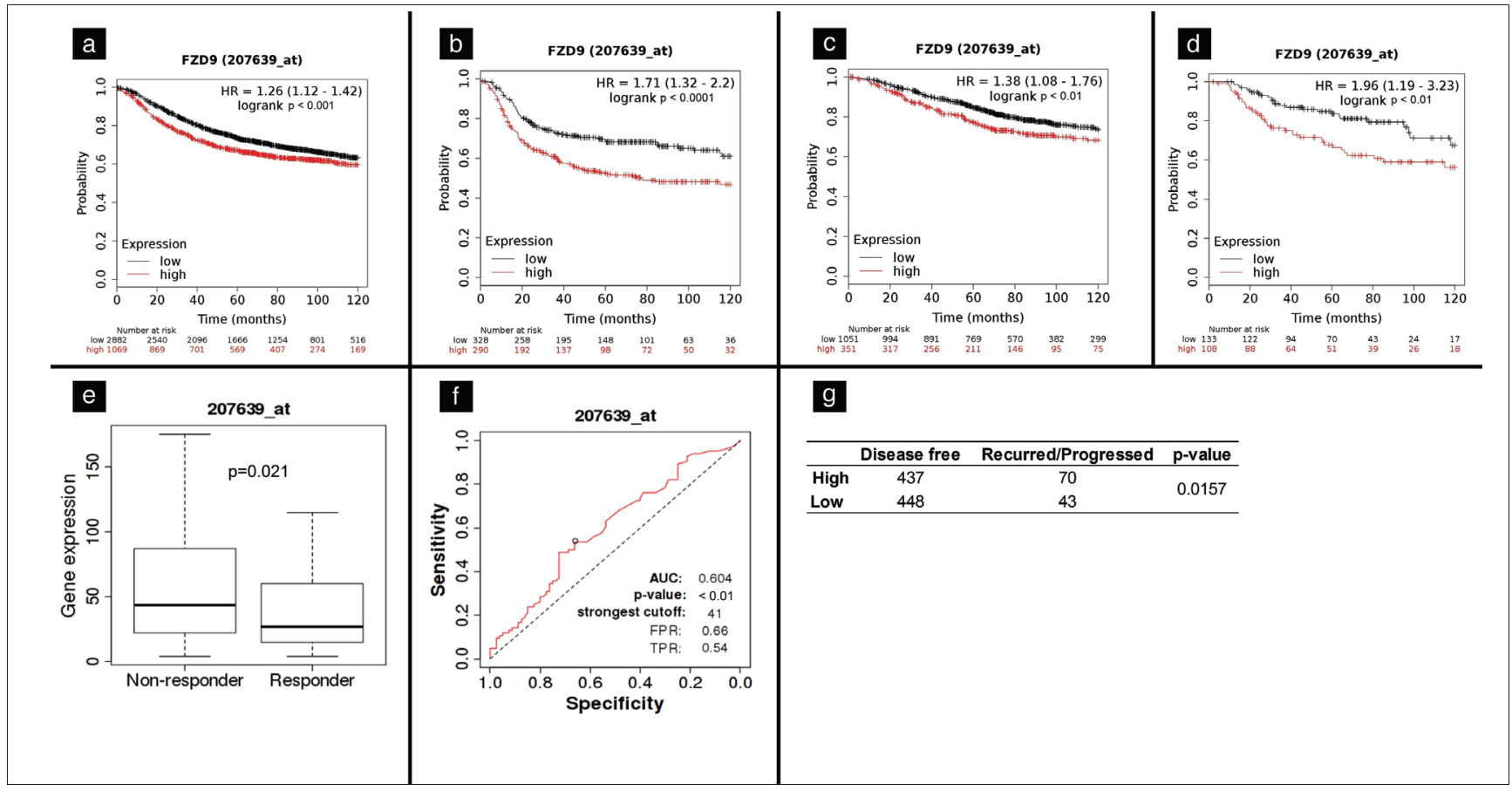

Figure 7. The expression pattern of FZD9 only in patients treated with chemotherapy. Relapse-free survival showing a) all tumor subtypes and b) basal subtypes. Overall survival analysis c) considering all subtypes of breast tumors and d) basal tumors; e) Relapse-free survival at 5 years between responders and non-responders to chemotherapy in TNBC patients; f) Roc curve for high and low FZD9 levels in responders and non-responders to chemotherapy. Area Under the Curve (AUC), True Positive Rate (TPR), and lower False Positive Rate (FPR); g) Association between FDZ9 variables and tumor recurrence. All survival curves were obtained on the KM Plotter. Roc curve and responder patients were obtained on the ROC Plotter platform. Recurred and disease-free statuses were obtained on clinical information using the Firehose from cBioPortal database 
The FZD family of receptors is the main mediator of Wnt signaling and consists of 10 members in humans (FZD1-FZD10), some of which have been proposed to be overexpressed in several tumor tissues $(23,24)$.

FZD9 functions as a molecular transmembrane signaling receptor, which has a $G$ protein-coupled receptor activity and functions in relation to Wnt-protein binding and protein homodimerization (25, 26). FZD9 and other FZD family members can be potentially used in new therapeutic strategies such as antibody-based ones and interfering molecule inhibitors, among many others (27). Herein, we found that FZD9 is differentially expressed with a highly significantly adjusted p-value in a cohort of 198 patients with TNBC compared to 67 patients with n-TNBC. In addition, we noted significant associations between the high expression of FZD9 with the clinical pathological characteristics, such as worse survival and prognosis, in patients treated with chemotherapy.

FZD9 dysregulation has already been associated with several tumors. In a study, it was found to be downregulated in lung cancer cell lines in contrast to that in gastric cancer cell lines and osteosarcoma samples (28), wherein an upregulation was observed $(12,29)$. Benhaj et al. (30) demonstrated a redundancy in the expression of ligands, receptors, coreceptors, and transcription factors of the Wnt pathway, including FZD9, in six different BC cell lines.

The FZD9 expression was increased in colorectal cancer tissues than in normal tissues and expressed in hepatocellular carcinoma (HCC) cell line, but absent in normal fetal and adult liver tissues $(31,32)$. Elsewhere, FZD9 knockdown reduced the cyclin D1 levels, migration, and cell proliferation in HCC cells (32). In contrast, non-small cell lung cancer cells with ectopic expression of Wnt7a/Fzd9 showed an increase in the PPARy activity and inhibited the transformation of growth suggesting an anti-tumorigenic effect (33).

Through immunohistochemistry, Wang et al. (29) observed high c-Fos, Wnt2 and FZD9 staining in patients with initial stage osteosarcoma and an even higher increase in the expressions of these proteins in more advanced tumors. The overexpression of c-Fos, Wnt2, and Fzd9 in the MG63 cell line compared to that in the normal cell line hFOB1.19 was observed in in vitro models, and the knockdown with iRNA against c-Fos resulted in the inhibition of migration, invasion, and proliferation, which promoted an increase in the MG63 cells. In addition, c-Fos knockdown reduced the Wnt2 and FZD9 expression. However, the hypothesis of direct interaction of c-Fos with Wnt2 and Fzd 9 was disregarded after conducting an immunoprecipitation assay (29). In our study, we noted a low negative correlation between FZD9 and Wnt2, which suggested that Wnt2 may not be the main mechanism of activation of Fzd9 in TNBC.

As reported by Karasawa et al. (34), the increase in the rat version of Fzd9, Rfz9, can recruit Dvl-1 and Axin, thus inducing the accumulation of cytoplasmic $\beta$-catenin, which results in TCF transcription activity. In the same study, the authors identified that Wnt2 alone can induce an increase in the $\beta$-catenin levels, although its activity in the nucleus remains without significant changes. On the other hand, the co-transfection of both Rfz 9 and Wnt2 leads to an increase in the concentration of $\beta$-catenin, which also sharply increases the TCF transcriptional activity (34). Therefore, we can speculate that Fzd9 in TNBC patients may act independently from Wnt2. However,
Wellenstein et al. (35) suggests that tumors harboring mutations or loss in p53 exhibit an increase in the Fzd9 expression, which predisposes to metastasis by a mechanism involving Wnt signaling and systemic inflammation. In addition to corroborating with our data that suggests association of the p53 mutational status with increased Fzd9 expression, the authors suggested that Fzd9 is one of the receptors of the Wnt pathway that can initiate a crosstalk between tumor cells and immune cells present in the tumor environment.

Cho et al. (20) analyzed a cohort of 184 patients with rectal cancer and divided them in two groups: good and poor responders. Initially, they used a group of patients labeled as training set and analyzed the genes that were differentially expressed in both the good and poor responder groups. This approach created a multigenic panel composed of eight genes, including FZD9, which were related to proliferation, cell cycle, tumor progression and development, and response to radiotherapy. Among the responders, low levels of FZD9 were observed (20). Herein, not only were high levels of FZD9 associated with worse OS and RFS but also with worse survival when stratifying for patients receiving chemotherapy. In agreement with Cho et al.'s (20) finding, we also noted that low levels of FZD9 are associated with a positive drug response. In addition, the ROC analysis of FZD9 revealed an AUC value that was extremely similar to HER2 (0.629), which is a classical well-established predictive biomarker in BC (19). Taken together with the findings of Cho et al. (20) and Fekete and Györffy (19), our data indicate that FZD9 can play an important role in the mechanism of drug resistance.

Zhang et al. (36) evaluated a cohort of 35 adult patients with cerebral cancer in addition to 10 normal individuals. Immunohistochemistry staining revealed a crescent level according to the histological tumor levels, among which grade IV showed the highest staining for FZD9. In addition, a positive correlation with the proliferation marker Ki67 was recorded (36). Similar to their findings, we observed an increase in the FZD9 mRNA levels in grade III patients, although this increase was not progressive, such as the ones reported by the investigators.

Taking together with other studies, our data strongly suggests that FZD9 is a promising biomarker and a therapeutic target for patients with TNBC, which can aid in the identification of tumor grades and prognosis. Collectively, our analysis was highly efficient for the screening of candidate genes and laid strong foundations for further in vitro and in vivo studies, which are necessary to consolidate these findings and apply them in the context of translational medicine.

It is important to highlight that in silico and data mining analysis can have considerable limitations. For instance, some platforms do not allow free access or manipulation and often have small cohorts, such as the TNBC patients categorized in the groups of responders and nonresponders to chemotherapy and the protein expression databases to confirm the relationship between mRNA and translated protein levels. This aspect often leads to not very robust results. Nonetheless, these analyses are highly efficient for the screening of candidate genes and for the further application of more complex approaches such as in vitro and in vivo assays.

Ethics Committee Approval: Ethics committee approval was not requested for this study.

Informed Consent: Informed consent was not requested for this study. 
Peer-review: Externally peer-reviewed.

\section{Authorship Contributions}

Concept: D.R.B., M.P.F.C.; Design: D.R.B., M.P.F.C.; Data Collection or Processing: D.R.B., M.P.F.C., A.P.P.M., J.M.R.S.L., J.J.D.S.; Analysis or Interpretation: D.R.B., M.P.F.C., A.P.P.M., J.M.R.S.L., R.A.S., R.C.C., J.J.D.S.; Literature Search: D.R.B., M.P.F.C., A.P.P.M., J.M.R.S.L., R.A.S., R.C.C., J.J.D.S., C.A.S.T.V., A.M.T.C.S.; Writing: D.R.B., M.P.F.C., A.P.P.M., J.M.R.S.L., R.A.S., R.C.C., J.J.D.S., C.A.S.T.V., A.M.T.C.S.

Conflict of Interest: The authors declare that there is no conflict of interest.

Financial Disclosure: This study was financed in part by the Coordenação de Aperfeiçoamento de Pessoal de Nível Superior - Brasil (CAPES) and Conselho Nacional de Desenvolvimento científico e tecnológico - Brasil (CNPq).

\section{References}

1. Ferlay J, Colombet M, Soerjomataram I, Mathers C, Parkin DM, Piñeros M, et al. Estimating the global cancer incidence and mortality in 2018: GLOBOCAN sources and methods. Int J Cancer 2019; 144: 1941-1953. (PMID: 30350310) [Crossref]

2. Perou CM, Sørile T, Eisen MB, Van de Rijn M, Jeffrey SS, Ress CA, et al. Molecular portraits of human breast tumours. Nature 2000; 406: $747-$ 752. (PMID: 23000897) [Crossref]

3. Sørlie T, Perou CM, Tibshirani R, Aas T, Geisler S, Johnsen H, et al. Gene expression patterns of breast carcinomas distinguish tumor subclasses with clinical implications. Proc Natl Acad Sci U S A 2001; 98: 1086910874. (PMID: 11553815) [Crossref]

4. Prat A, Perou C. Molecular classification of triple-negative tumors. Breast Cancer Res 2011; 13(Suppl 2): O2 (PMID: 21278442) [Crossref]

5. Collignon J, Lousberg L, Schroeder H, Jerusalem G. Triple-negative breast cancer: treatment challenges and solutions. 2016; 8: 93-107. (PMID: 27284266) [Crossref]

6. Gazinska P, Grigoriadis A, Brown JP, Millis RR, Mera A, Gillett CE, et al. Comparison of basal-like triple-negative breast cancer defined by morphology, immunohistochemistry and transcriptional profiles. Mod Pathol 2013; 26: 955-966. (PMID: 23392436) [Crossref]

7. Lehmann BD, Bauer JA, Chen X, Sanders ME, Chakravarthy AB, Shyr Y, et al. Identification of human triple-negative breast cancer subtypes and preclinical models for selection of targeted therapies. J Clin Invest 2011; 121: 2750-2767. (PMID: 21633166) [Crossref]

8. Duchartre Y, Kim YM, Kahn M. The Wnt signaling pathway in cancer. Crit Rev Oncol Hematol 2016; 99: 141-149. (PMID: 26775730) [Crossref]

9. Amin N, Vincan E. The Wnt signaling pathways and cell adhesion. Front Biosci (Landmark Ed) 2012; 17: 784-804. (PMID: 22201774) [Crossref]

10. Libro R, Bramanti P, Mazzon E. The role of the Wnt canonical signaling in neurodegenerative diseases. Life Sci 2016; 158: 78-88. (PMID: 27370940) [Crossref]

11. Polakis P. Wnt signaling in cancer. Cold Spring Harb Perspect Biol 2012; 4: a008052. (PMID: 22438566) [Crossref]

12. Kirikoshi H, Sekihara H, Katoh M. Expression profiles of 10 members of Frizzled gene family in human gastric cancer. Int J Oncol 2001; 19: $767-$ 771. (PMID: 11562753) [Crossref]

13. Burstein MD, Tsimelzon A, Poage GM, Covington KR, Contreras A, Fuqua SAW, et al. Comprehensive genomic analysis identifies novel subtypes and targets of triple-negative breast cancer. Clin Cancer Res 2015; 21: 1688-1698. (PMID: 25208879) [Crossref]

14. Jézéquel P, Campone M, Gouraud W, Guérin-Charbonnel C, Leux C, Ricolleau G, et al. Bc-GenExMiner: An easy-to-use online platform for gene prognostic analyses in breast cancer. Breast Cancer Res Treat 2012; 131: 765-775. (PMID: 21452023) [Crossref]

15. Jézéquel P, Frénel J-S, Campion L, Guérin-Charbonnel C, Gouraud W, Ricolleau G, et al. bc-GenExMiner 3.0: new mining module computes breast cancer gene expression correlation analyses. Database (Oxford) 2013; 2013: bas060. (PMID: 23325629) [Crossref]

16. Györffy B, Lanczky A, Eklund AC, Denkert C, Budczies J, Li Q, et al. An online survival analysis tool to rapidly assess the effect of 22,277 genes on breast cancer prognosis using microarray data of 1,809 patients Breast Cancer Res Treat 2010; 123: 725-731. (PMID: 20020197) [Crossref]

17. Cerami E, Gao J, Dogrusoz U, Gross BE, Sumer SO, Aksoy BA, et al. The cBio Cancer Genomics Portal: An open platform for exploring multidimensional cancer genomics data. Cancer Discov 2012; 2: 401404. (PMID: 22588877) [Crossref]

18. Gao J, Aksoy BA, Dogrusoz U, Dresdner G, Gross B, Sumer SO, et al. Integrative analysis of complex cancer genomics and clinical profiles using the cBioPortal. Sci Signal 2013; 6: pl1. (PMID: 23550210) [Crossref]

19. Fekete JT, Györffy B. ROCplot.org: Validating predictive biomarkers of chemotherapy/hormonal therapy/anti-HER2 therapy using transcriptomic data of 3,104 breast cancer patients. Int J Cancer 2020; 145: 3140-3151. (PMID: 31020993) [Crossref]

20. Cho E, Park IJ, Yeom SS, Hong SM, Lee JB, Kim YW, et al. A multigene model for predicting tumor responsiveness after preoperative chemoradiotherapy for rectal cancer. Int J Radiat Oncol 2019; 105: 834842. (PMID: 31419511) [Crossref]

21. Pohl SG, Brook N, Agostino M, Arfuso F, Kumar AP, Dharmarajan A. Wnt signaling in triple-negative breast cancer. Oncogenesis 2017; 6: e310. (PMID: 28368389) [Crossref]

22. Zhan T, Rindtorff N, Boutros M. Wnt signaling in cancer. Oncogene 2017; 36: 1461-1473. (PMID: 27617575) [Crossref]

23. Yang L, Wu X, Wang Y, Zhang K, Wu J, Yuan YC, et al. FZD7 has a critical role in cell proliferation in triple negative breast cancer. Oncogene 2011; 30: 4437-4446. (PMID: 21532620) [Crossref]

24. Ueno K, Hirata H, Hinoda Y, Dahiya R. Frizzled homolog proteins, microRNAs and Wnt signaling in cancer. Int J Cancer 2013; 132: 17311740. (PMID: 22833265) [Crossref]

25. Katoh M. WNT signaling in stem cell biology and regenerative medicine. Curr Drug Targets 2008; 9: 565-570. (PMID: 18673242) [Crossref]

26. Huang HC, Klein PS. The frizzled family: Receptor for multiple signal transduction pathways. Genome Biol 2004; 5: 234. (PMID: 15239825) [Crossref]

27. Zeng CM, Chen Z, Fu L. Frizzled receptors as potential therapeutic targets in human cancers. Int J Mol Sci 2018; 19: 1543. (PMID: 29789460) [Crossref]

28. Tennis MA, New ML, McArthur DG, Merrick DT, Dwyer-Nield LD, Keith RL. Prostacyclin reverses the cigarette smoke-induced decrease in pulmonary Frizzled 9 expression through MIR-31. Sci Rep 2016; 6: 28519. (PMID: 27339092) [Crossref]

29. Wang Q, Liu H, Wang Q, Zhou F, Liu Y, Zhang Y, et al. Involvement of c-Fos in cell proliferation, migration, and invasion in osteosarcoma cells accompanied by altered expression of Wnt2 and Fzd9. PLoS One 2017; 12: 1-16. (PMID: 28665975) [Crossref]

30. Benhaj K, Akcali KC, Ozturk M. Redundant expression of canonical Wnt ligands in human breast cancer cell lines. Oncol Rep 2006; 15: 701-707. (PMID: 16465433) [Crossref]

31. Nagayama S, Yamada E, Kohno Y, Aoyama T, Fukukawa C, Kubo H, et al. Inverse correlation of the up-regulation of FZD10 expression and the activation of $\beta$-catenin in synchronous colorectal tumors. Cancer Sci 2009; 100: 405-412. (PMID: 19134005) [Crossref] 


\section{Eur J Breast Health 2021; 17(1): 42-52}

32. Fujimoto T, Tomizawa M, Yokosuka O. SiRNA of Frizzled-9 suppresses proliferation and motility of hepatoma cells. Int J Oncol 2009; 35: 861866. (PMID: 19724923) [Crossref]

33. Winn RA, Van Scoyk M, Hammond M, Rodriguez K, Crossno JT, Heasley LE, et al. Antitumorigenic effect of Wnt 7a and Fzd 9 in nonsmall cell lung cancer cells is mediated through ERK-5-dependent activation of peroxisome proliferator-activated receptor $\gamma$. J Biol Chem 2006; 281: 26943-26950. (PMID: 16835228) [Crossref]

34. Karasawa T, Yokokura H, Kitajewski J, Lombroso PJ. Frizzled-9 is activated by Wnt- 2 and functions in Wnt/B-catenin signaling. J Biol Chem 2020; 277: 37479-37486. (PMID: 12138115) [Crossref]
35. Wellenstein MD, Coffelt SB, Duits DEM, van Miltenburg MH, Slagter $\mathrm{M}$, de Rink I, et al. Loss of p53 triggers WNT-dependent systemic inflammation to drive breast cancer metastasis. Nature 2020; 572: 538542. (PMID: 31367040) [Crossref]

36. Zhang Z, Schittenhelm J, Guo K, Bühring HJ, Trautmann K, Meyermann $\mathrm{R}$, et al. Upregulation of frizzled 9 in astrocytomas. Neuropathol Appl Neurobiol 2006; 32: 615-624. (PMID: 17083476) [Crossref] 